\title{
Robust Remote Sensing Still Image Coding With Low Memory Requirements
}

\author{
Arthur L. A. da Cunha ${ }^{\dagger}$, Weiler A. Finamore ${ }^{\dagger}$, and Eduardo A. B. da Silva ${ }^{\dagger \dagger}$ \\ † Pontifícia Universidade Católica do Rio de Janeiro \\ Centro de Estudos em Telecomunicações \\ Rua Marquês de São Vicente, 255 (CETUC) \\ 22453-900, Rio de Janeiro, RJ - Brazil \\ \{arthur, weiler\}@cetuc.puc-rio.br \\ †† Universidade Federal do Rio de Janeiro \\ $\mathrm{PEE} / \mathrm{COPPE} / \mathrm{DEL} / \mathrm{EE}$ \\ Caixa Postal 68504 \\ 21945-970, Rio de Janeiro, RJ - Brazil \\ eduardo@lps.ufrj.br
}

\begin{abstract}
An scheme for the efficient transmission of remote sensing satellite (RSS) images is presented in this paper. A joint source/channel coding solution was used to deal with the conflicting issues of data compression, to reduce the amount of data to be sent, and data protection, to cope with errors introduced by the channel, issues further burdened by the stringent low complexity and low memory requirements brought by the need for on-board compression. Results are presented showing that the proposed scheme attains good PSNR performance while still maintaining low complexity.
\end{abstract}

\section{INTRODUCTION}

Remote sensing imagery produces large amounts of data that often needs to be lossy compressed and reliably transmitted over a bandlimited channel. Ordinarily, compression and data protection are carried out separately, namely, in tandem approach. Such technique is motivated by Shannon's separation principle which is an asymptotic result that holds when encoding large blocks of data. This requires an overwhelming complexity often not available, for example, in remote sensing satellite circuitry. An alternative to the tandem scheme, when delay and complexity are somewhat constrained, is joint source/channel coding in which compression and protection is accomplished jointly in a single step. A classical example of source/channel coding is the channel-optimized vector quantizer (COVQ) [1] and its scalar version, the channel-optimized scalar quantizer (COSQ) [2]. The reproduction codevectors are designed taking into account wrongly received quantizer indices. In such case protection against channel errors is obtained by a tradeoff between quantizer performance and Euclidean distance between the various codevectors. Although for memoryless sources the COVQ would perform better for moderate block length [1], the trouble with the COVQ is that indices received in error cause annoying impulsive artifacts in the reconstructed images. Such artifacts are unacceptable for most remote sensing applications.

Several schemes for compressing images to be transmitted over noisy channels have been proposed recently. In [3] the impulsive noise problem is circumvented by a nonlinear all-pass filter preceding the COSQ. The all-pass filtering improves the quantizer performance and also spreads the impulsive artifacts producing images with better visual quality. One of the best published $S N R$ (Signal-to-Noise Ratio) performance results were obtained with the tandem scheme (compression followed by forward error correction) proposed by Sherwood and Zeger in [4] — the bitstream output by a SPIHT encoder is, before transmission, segmented into fixed-length packets which are then protected with check-sums and punctured convolutional codes. Sherwood and Zeger scheme has a non-negligible probability of incomplete (or catastrophic) decoding and usually would perform poorer under channel mismatch condition. Since it relies on a wavelet decomposition, it requires also a large amount of memory. Typically $512 \times 512$ pixels of image data has to be stored in order to compute the wavelet transform - the performance worsens substantially if applied to blocks of images with smaller sizes. In addition, it should be stressed that the encoding process on tandem scheme usually introduces a long coding delay and adds extra complexity.

The alternative to tandem coding proposed in this paper uses a joint source/channel coding scheme with a high performance Generalized Lapped Bi-orthogonal Transform (GLBT) [5]. The small blocks into which the image is subdivided are transformed and the resulting transform coefficients are scrambled by means of an all-pass nonlinear filter, the output of which is quantized with a bank of channel-optimized scalar quantizers (one for each coefficient), similarly to the procedure presented in [3]. The sequence at the output of the quantizers are then encoded with rates as determined by a fast and efficient steepest descent bit allocation algorithm. The paper is organized as follows: in section we present the algorithm and its basics building blocks. Results and Conclusion are presented in sections and respectively.

\section{Proposed Scheme structure}

Fig. 1 displays the first step of our coding scheme. Encoding is accomplished in block-of-lines acquired on the fly by the satellite image sensors, each containing $n$ image lines. Each block-of-lines, after being captured, is segmented into $8 \times 8$ blocks. These blocks are transformed by the LT linear operator. Once the buffer is full (i.e, contains a block-of-lines), the variances of coefficients within the same subband are estimated and the coefficients normalized. The variances are later used in the allocation strategy described in the next section. The algorithm forms sequences of coefficients within the same subband and these are robust quantized according to the bit rate stipulated in the allocation procedure. The variances together with the 
allocation matrix is transmitted as side-information. Since this side information represents a small amount of data a simple channel coding scheme can be used to protect this information (for example, for typical lines of more than 3000 pixels, the coded side information represents $\sim 0.1 \mathrm{bpp}$ in the worst case). After the current buffer is encoded, the buffer is filled again and the encoding algorithm restarted. Decoding each block-of-lines is simply the reverse process. After the side information have been decoded, the de-normalized quantizer centroids obtained from the indices are de-scrambled and fed into the LT reconstruction module. Care must be taken in the boundaries since, in order to compute an LT of the first 8 lines of a buffer one needs the last 8 lines of the previous buffer.

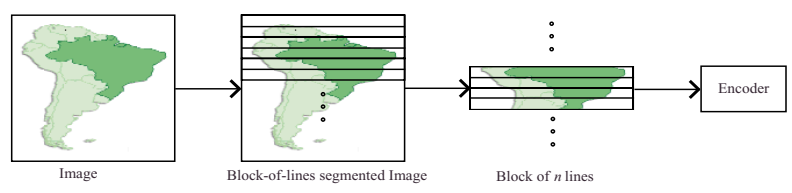

Fig. 1. Illustration of the proposed scheme.

\section{A. Lapped Transforms}

Lapped Transform were first introduced to reduce blocking artifacts inherent to block transform such as the DCT. The LT analysis stage can be understood as a postprocessing over the DCT coefficients of adjacent blocks, which is carried out in a way that the corresponding transform basis functions extend across block boundaries thus mitigating the blocking or Gibbs phenomenon. A popular LT for image compression is the Lapped Orthogonal Transform (LOT) introduced by Malvar and Staelin in [6]. While the LOT reduce significantly the Gibbs phenomenon it does not eliminate it completely.

Recently, research efforts have lead to a wide variety of new LT's filters with comparable performance to the wavelet popular 9/7 filters [5], [7]. For example the Generalized Bi-orthogonal Transform introduced in [5] can attain coding gains of up to $9.96 \mathrm{~dB}$. In constract,a three-stage wavelet decomposition with $9 / 7$ filters has a coding gain of $9.45 \mathrm{~dB}$. Throughout this work we will be using the $8 \times 16$ Generalized Bi-orthogonal transform presented in [5], [8] for its good performance versus complexity tradeoff.

\section{B. Bit Allocation}

After the coefficients are normalized bit allocation takes place. The problem of bit allocation for $N$ independent sources can be understood as the selection of a set $\mathcal{R}=\left\{r_{1}, r_{2}, \ldots, r_{N}\right\}$ of bit rates that minimizes the overall distortion

$$
D(\mathcal{R})=\frac{1}{N} \sum_{i=1}^{N} d_{i}\left(r_{i}\right)
$$

constrained to $\sum_{i=1}^{N} r_{i} \leq \bar{r} N$. In (1) $d_{i}\left(r_{i}\right)$ is the average distortion incurred by the $i$-th quantizer and $\bar{r}$ denotes the target average bit rate. In order to efficiently solve the bit allocation problem we have followed the results presented in [9]. A similar allocation strategy based on the steepest descent was employed. This algorithm which is specially suitable for channel-optimized quantizers, can be summarized as follows:

1. Set $k=\bar{r} N ;$ Set $r_{i}=0, i=1, \ldots, N$

2 . Set $k=k-1$; find $i_{k}$ which satisfy

$$
\Delta_{i_{k}}\left(r_{i_{k}}\right)=\max _{i=\{1, \ldots, N\}} d_{i}\left(r_{i}\right)-d_{i}\left(r_{i}+1\right)
$$

3. Set $r_{i_{k}}=r_{i_{k}}+1$. if $k=0$ stop; else go to step 2 .

Since we are dealing with matrices of coefficients the index $i$ in the algorithm actually is a double index $i, j-$ we dropped the $j$ for the sake of clarity. The search can be speeded by testing only the adjacent coefficients in the diagonal direction, i.e, for each pair $(i, j)$ which has assigned to it at least one bit, test only $(i, j+1),(i+1, j)$ and $(i+1, j+1)$. We verified in our experiments that the algorithm produces results very close to an optimal allocation. Furthermore, with pre-computed distortion-rate pairs the allocation is accomplished with a minimal number of operations and indeed runs very fast. As a comment, we note that when the channel is noisy the algorithm tend to protect more important coefficients such as the DC, thus assigning more bits for such coefficients.

\section{Robust Quantization}

In order to cope with the occasional errors introduced by the channel we have used the COSQ preceded by a phase scrambler (all-pass filtering) akin to that of [3]. In it, for each sequence of coefficients, a fast Fourier transform (FFT) is calculated and separated into magnitude and phase. A generated pseudo-noise sequence or, more especifically, an $m$-sequence is then added to the phase and the inverse FFT of the resulting sequence calculated. Since the magnitude remains the same, the scrambler can be seen as non-linear all-pass filter. The decoder follows the same procedure subtracting the $m$-sequence re-generated at the receiver end.

The scrambler has two interesting actions:(1) It preforms source histogram re-shaping and (2) It spreads the impulsive noise caused by wrong received quantizer indices. It can be shown that for Generalized Gaussian sources with shape parameter less that 2.0, such as LT coefficients, the scrambler re-shpaping in fact increases the shape parameter of the source in such a way that the new histogram is a near Gaussian one. As a result, the quantizer performance increases since the larger the shape-parameter, the better a scalar quantizer will perform [3]. Scrambling the sequences also brings a dramatic perceptual advantage since the impulsive noise is virtually eliminated. Finnally, we point out that the phase scrambler can be used with any quantizer, as for example, the trellis-coded quantizer used in [10]. We chose to use COSQ given its low complexity in comparison to the channel-optimized TCQ of [10]. 


\section{Results}

Images that simulates a $180 \mathrm{~m}$ ground resolution images, to be captured by a (yet to be launched) LEO RSS provided by the Brazilian Space Agency (INPE), were used as a test bed for the proposed scheme. The results obtained when compressing RSS images are very promising. Tab .1 shows the results obtained for a binary symmetric channel with bit error probability $P_{e}=10^{-3}$ and several compression rates and memory sizes (number of lines).

TABLE I

PSNR FOR A TYPICAL RSS $1024 \times 1024$ IMAGE FOR VARIOUS COMPRESSION RATIOS AND MEMORY SIZES.

\begin{tabular}{|c|c|c|c|c|}
\hline $\begin{array}{c}\text { Comp. } \\
\text { Ratio }\end{array}$ & $\begin{array}{c}\text { Proposed } \\
\text { 32 lines }\end{array}$ & $\begin{array}{c}\text { Proposed } \\
\text { 64 lines }\end{array}$ & $\begin{array}{c}\text { Proposed } \\
\text { 256 lines }\end{array}$ & $\begin{array}{c}\text { Scheme of [4] } \\
1024 \times 1024\end{array}$ \\
\hline \hline $1: 16$ & 37.33 & 37.64 & 37.75 & 39.32 \\
\hline $1: 10$ & 39.10 & 39.29 & 39.45 & 41.20 \\
\hline $1: 8$ & 40.21 & 40.35 & 40.36 & 42.28 \\
\hline
\end{tabular}

Compared to the scheme proposed by Sherwood and Zeger our scheme is inferior as far as peak-SNR (PSNR) performance is concerned but has the advantage of being less complex and less memory demanding. It also does not suffer from the incomplete decoding (synchronization loss) problem inherent to the SPIHT compressor. Fig. 2 displays typical decoded images and the corresponding error images (equalized for better visualization), for various bit error probabilities. As can be seen, graceful degradation is obtained as the probability of error increases. Moreover, for RSS applications where losses can be tolerated, even the image transmitted with $P_{e}=10^{-2}$ has acceptable quality. This is mainly due to the phase-scrambler which reduces dramatically the perceptual effect of the impulsive noise.

\section{Conclusion and Further Directions}

We have proposed an scheme for efficient robust compression of RSS images. Our scheme is based on highly efficient LT, and an optimal steepest descent bit allocation algorithm and a phase scrambler used to deal with the channel noise. In comparison to the scheme proposed by Fischer [3] with comparable complexity and memory requirement, our scheme besides being less storage demanding, yields better results (natural images only were used in this comparison). Currently we are investigating ways of handling multi-spectral imagery, as well as classification algorithms to enhance the performance of the algorithm, specially for RSS images featuring high non-stationarity.

\section{REFERENCES}

[1] N. Farvardin, "A study of vector quantization for noisy channels," IEEE Trans. on Inf. Theory, vol. 36, pp. 799-809, July 1990.

[2] N. Farvardin and V. Vaishampayan, "Optimal quantizer design for noisy channels: An approach to combined source-channel coding," IEEE Trans. Inf. Theory, vol. 33, pp. 827-838, November 1987.

[3] Q. Chen and T. R. Fischer, "Image coding using robust quantization for noisy digital transmission," IEEE Trans. on Img. Proc., vol. 7, pp. 496-505, April 1998.

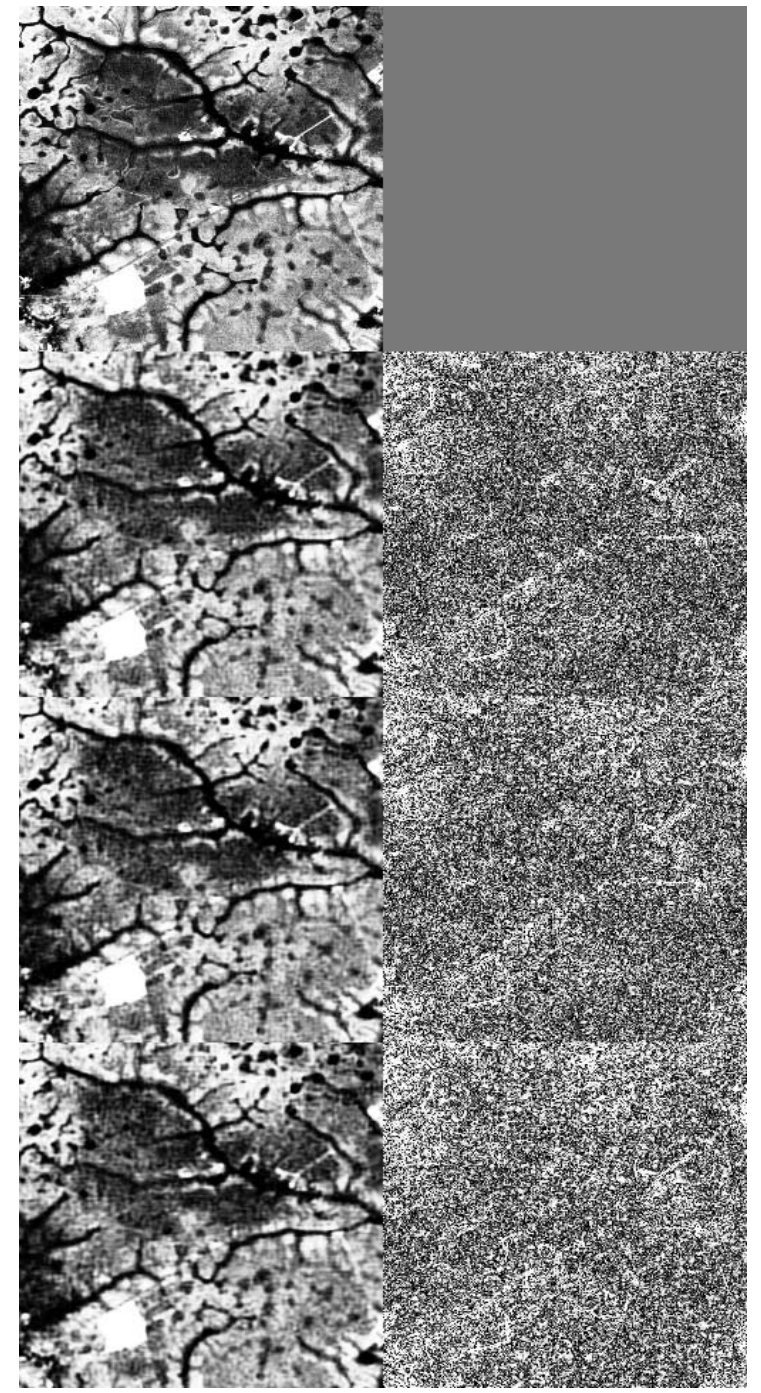

Fig. 2. Example of typical decoded images at $0.5 \mathrm{bpp}, 64$ lines. From top to bottom: original, $P_{e}=10^{-4}-\mathrm{PNSR}=37.89 \mathrm{~dB}, P_{e}=$ $10^{-3}-\mathrm{PSNR}=37.57 \mathrm{~dB}, P_{e}=10^{-2}-\mathrm{PSNR}=36.78 \mathrm{~dB}$.

[4] P. G. Sherwood and K. Zeger, "Progressive image coding on noisy channels," Proc. IEEE Data Compress. Conf., pp. 72-81, March 1997.

[5] T. D. Tran, R. L. de Queiroz, and T. Q. Nguyen, "Linear phase perfect reconstruction filter bank: lattice structure, design, and application in image coding," IEEE Trans. on Sig. Proc., vol. 48, pp. 133-147, Jan. 2000.

[6] H. S. Malvar and D. H. Staelin, "The lot: Transform coding without blocking effects," IEEE Trans. on Signal Processing, vol. 37, pp. pp. 553-559., April 1989.

[7] R. L. de Queiroz, T. Q. Nguyen, and K. R. Rao, "The GenLOT: Generalized linear-phase lapped orthogonal transforms," IEEE Trans. on Sig. Proc., vol. 44, pp. 497-507, April 1996.

[8] R. L. de Queiroz and T. D. Tran, "Lapped transforms for image compression," in The Handbook on Transforms and Data Compression (K. R. Rao and P. Yip, eds.), ch. 5, CRC Press, Oct. 2000 .

[9] V. Vaishampayan and N. Farvardin, "Optimal block cosine transform image coding for noisy channels," IEEE Trans. on Commun., vol. 38, pp. 327-336, March 1990.

[10] T.-T. Lam, G. P. Abousleman, and L. J. Karam, "Image coding with robust channel-optimized trellis-coded quantization," IEEE JSAC, vol. 18, pp. 940-951, June 2000. 